\title{
Aktuelle Erkenntnisse zur Eigenfett Transplantation anhand der neuen Leitlinie „Autologe Fetttransplantation“"
}

\author{
Current Perceptions of Lipofilling on the Basis of the New Guideline on \\ "Autologous Fat Grafting"
}

Autor

Institute
L. Prantl ${ }^{1 *}$, H. O. Rennekampff ${ }^{*}$, R. E. Giunta ${ }^{3}$, Y. Harder ${ }^{4}$, D. von Heimburg ${ }^{5}$, N. Heine ${ }^{6}$, C. Herold U. Kneser ${ }^{8}$, F. Lampert ${ }^{9}$, H. G. Machens ${ }^{10}$, U. Mirastschijski ${ }^{11}$, D. Müller ${ }^{12}$, N. Pallua ${ }^{13}$, T. Schantz ${ }^{14}$, A. Schönborn ${ }^{15}$, K. Ueberreiter ${ }^{16}$, CH. Witzel ${ }^{17}$, G. Bull ${ }^{18}$, D. Rezek ${ }^{19}$, G. Sattler ${ }^{20}$, P. M. Vogt $^{21}$, R. E. Horch 22

Die Institutsangaben sind am Ende des Beitrags gelistet

\section{Schlüsselwörter \\ - Eigenfett Transplantation \\ - Leitlinie \\ - DGPRÄC \\ - Neue Erkenntnisse}

\section{Key words}

- Fat Grafting

- Guideline

- German Society of Plastic, Reconstructive and Aesthetic Surgery

- New Insights eingereicht 13.9.2016

akzeptiert 16.9.2016

\section{Bibliografie}

DOI http://dx.doi.org/

10.1055/s-0042-117635

Online-Publikation: 10.11.2016

Handchir Mikrochir Plast Chir

2016; 48: 330-336

(c) Georg Thieme Verlag KG

Stuttgart · New York

ISSN 0722-1819

\section{Korrespondenzadresse}

Prof. Dr. Lukas Prantl

Abteilung für Plastische,

Hand- und Wiederherstellungs-

chirurgie,

Universität Regensburg,

Regensburg

Franz-Josef-Strauß-Allee 11

93042 Regensburg

lukas.prantl@klinik.

uni-regensburg.de

\section{Zusammenfassung}

$\nabla$

Hintergrund: Die autologe Fetttransplantation ist eine in letzter Zeit zunehmend verbreitete operative Prozedur und umfasst die Entnahme, Bearbeitung und Transplantation von Fettgewebe sowie die fachgerechte Nachsorge. Dieser operative Eingriff ist ein Verfahren, das sowohl nach traumatischem, krankheits- oder altersbedingtem Volumenverlust der Weichteile als auch zur Weichteilaugmentation eingesetzt werden kann. Aufgrund der zunehmenden Anwendung aber noch bisher wenig definierten Variablen in der Fettgewinnung, Indikationsstellung und Art der Transplantation in der rekonstruktiven und ästhetischen Chirurgie besteht ein großes Interesse an der Entwicklung einer Leitlinie.

Methodik: In einer Konsensuskonferenz im Rahmen eines nominalen Gruppenprozesses der anwendenden Fachgesellschaften wurden alle im Delphi-Verfahren strittigen Punkte diskutiert und mit starken Konsens (>95\%) beschlossen. Die Literatur der letzten 10 Jahre in den gängigen medizinischen Informationsportalen und Datenbanken wurde abgefragt, studiert und unter Berücksichtigung auch wichtiger älterer Arbeiten entscheidende Aussagen der Leitlinie belegt.

Ergebnisse: Unter Einschluss aller relevanten Fachgesellschaften wurden hinsichtlich nachfolgender Punkte konsensbasierte Empfehlungen in Form dieser Leitlinie formuliert. 1. Definition, 2. Indikation/Kontraindikation, 3. Präoperative Maßnahmen, 4. Entnahmetechniken, 5. Bearbeitungstechniken, 6. Transplantation, 7. Nachsorge, 8. Aufbewahrung und Lagerung, 9. Effizienz, 10. Dokumentation, 11. Bewertung der Sicherheit.

Zusammenfassung: Klare Indikationsstellung und fachliche Expertise sind Grundvoraussetzung um die autologe Fetttransplantation durchzuführen. Der Erfolg dieses operativen Eingriffs

\section{Abstract \\ $\nabla$}

Introduction: Autologous fat transfer has recently become an increasingly popular surgical procedure and comprises harvesting, processing and transplantation of adipose tissue, as well as professional follow-up care. This method, as a surgical procedure, can be utilised for trauma-, disease- or age-related soft tissue volume deficits and soft tissue augmentation. As usage is increasing, but the variables of fat harvest, specific indications and fashion of fat transfer are poorly defined, there is a great demand for development of a guideline in the field of reconstructive and aesthetic surgery.

Methods: All relevant points were discussed within the scope of a consensus conference including a nominal group process of all societies involved in the procedure and ratified with a strong consensus $(>95 \%)$. Literature from the standard medical databases over the last 10 years was retrieved, studied and specific guidelines were concluded.

Results: Consensus was achieved among all professionals involved on the following points: 1 . definition 2 . indication/contraindication, 3. preoperative measures 4 . donor sites 5. techniques of processing 6 . transplantation 7. follow-up care 8 . storage 9 . efficacy 10 . documentation 11. evaluation of patient safety.

Conclusion: Definite indications and professional expertise are paramount for autologous fat tissue transfer. Successful transfers are based on the use of correct methods as well as specific instruments and materials. Autologous adipose tissue transplantation is considered to be a safe procedure in reconstructive and aesthetic surgery, due to the low rate of postoperative complications and sequelae. 
ist abhängig vom Einsatz der richtigen Methoden sowie der speziellen Instrumente und Materialen. Aufgrund der niedrigen Rate an postoperativen Komplikationen und Spätfolgen ist die autologe Fetttransplantation als sicheres Verfahren in der rekonstruktiven und ästhetischen Medizin anzusehen.

Teile dieses Artikels sind wörtlich aus der Leitlinie entnommen ${ }^{1}$

\section{Einleitung}

\section{$\nabla$}

Die autologe Fetttransplantation ist ein zunehmend angewandtes Verfahren in der rekonstruktiven und ästhetischen Chirurgie. Daraus ergeben sich Anforderungen an die Versorgungsqualität mit qualifizierter Behandlung. Um diesen Anforderungen nachzukommen wurde eine Leitlinie zur Durchführung dieser Maßnahme durch einen interdisziplinären Expertenkonsens formuliert. Die Gewährleistung der Patientensicherheit während der Anwendung der autologen Fetttransplantation sowie die Optimierung der gesamten Therapie, der Lebensqualität der Patienten und des Kostenaufwandes im prä-, peri- und postoperativen Management sind als vorrangige Ziele dieser Leitlinie zu nennen.

\section{Methodik}

Die Leitlinie zur „Autologen Fetttransplantation“ wurde von der Deutschen Gesellschaft der Plastischen, Rekonstruktiven und Ästhetischen Chirurgen (DGPRÄC) unter der Koordination von Prof. Dr. Hans-Oliver Rennekampff unter der Mitarbeit der Deutschen Dermatologischen Gesellschaft (DDG), der Deutschen Gesellschaft für Mund-, Kiefer- und Gesichtschirurgie (DGMKG) und der Deutschen Gesellschaft für Gynäkologie und Geburtshilfe (DGGG) erstellt. Die Projektanmeldung der Leitlinie mit einer geplanten Entwicklungsstufe S2k bei der Arbeitsgemeinschaft der Wissenschaftlichen Medizinischen Fachgesellschaften e. V. (AWMF) erfolgte im November 2010. Unter Moderation der AWMF fand im August 2014 die Konsensuskonferenz statt, um eine Abstimmung der Leitlinie im Hinblick auf Diagnostik und Therapie unter Einbeziehung aller an Diagnostik und Therapie beteiligten Fachgesellschaften zu erreichen. Alle im Delphi-Verfahren nach der Konsensuskonferenz strittigen Punkte wurden einzeln diskutiert und anschließend mit starkem Konsens ( $>95 \%$ ) beschlossen. Eine systematische Literaturanalyse und Evidenzbewertung erfolgte durch die Experten, wobei die Literatur der letzten zehn Jahre über medline, pubmed, Silver Platter, EMBASE und cochrane abgefragt, studiert und unter Berücksichtigung auch wichtiger älterer Arbeiten maßgebliche Aussagen der Leitlinie belegt wurden. Bei der Leitlinien-Entwicklung

\footnotetext{
${ }^{1}$ Leitlinienkoordination: Prof. Dr. Hans-Oliver Rennekampff, Leverkusen, Steuergruppe (DGPRÄC): Prof. Dr. Riccardo Giunta, München Prof. Dr. Dennis von Heimburg, Frankfurt Prof. Dr. Ulrich Kneser, Ludwigshafen Dr. Florian Lampert, Freiburg Prof. Dr. Hans-Günther Machens, München Prof. Dr. Norbert Pallua, Aachen Prof. Dr. Lukas Prantl, Regensburg Dr. Klaus Ueberreiter, Birkenwerder Prof. Dr. Peter M. Vogt, HannoverAutoren: Prof. Dr. Riccardo Giunta, München Prof. Dr. Yves Harder, Viganello Prof. Dr. Dennis von Heimburg, Frankfurt Dr. Norbert Heine, Regensburg Priv.-Doz. Christian Herold, Hameln Prof. Dr. Ulrich Kneser, Ludwigshafen Dr. Florian Lampert, Freiburg Prof. Dr. Hans-Günther Machens, München Priv.-Doz. Dr. Dr. Ursula Mirastschijski, Bremen Priv.-Doz. Dr. Daniel-Felix Müller,München Prof. Dr. Lukas Prantl, Regensburg Dr. Jan-Thorsten Schantz, München Dr. Alexander Schönborn, Potsdam Dr. Klaus Ueberreiter, Birkenwerder Dr. Christian Witzel, Berlin
}

wurden die Kriterien des Deutschen Instruments für Leitlinien (DELBI) berücksichtigt.

\section{Definition und Indikationsstellung \\ $\nabla$}

Die autologe Fetttransplantation ist eine operative Prozedur und umfasst die Entnahme, Bearbeitung und Transplantation von Fettgewebe sowie die fachgerechte Nachsorge. Dieser operative Eingriff ist häufig ein sekundär-rekonstruktives Verfahren, das sowohl nach traumatischem, krankheits- oder altersbedingtem Volumenverlust der Weichteile als auch zur Weichteilaugmentation eingesetzt werden kann. Zunehmend zeichnet sich über den eigentlichen Volumenersatz auch ein positiver Effekt auf Narben- und Hautqualität ab. Somit besteht zusätzlich meist eine Verbesserung der Lebensqualität als indirektes Therapieziel.

Die autologe Fetttransplantation als hochelektiver Eingriff fordert hinsichtlich der Patientensicherheit besonders strenge Maßstäbe. Ein nachvollziehbarer und realistisch erfüllbarer Patientenwunsch nach Rekonstruktion oder ästhetischer Verbesserung der Weichteilsituation sollte für die Indikationsstellung grundlegend sein. Relative und absolute Indikationen sowie Kontraindikationen sind in $\odot$ Tab. 1 und $\odot$ Tab. 2 aufgeführt, jedoch soll individuell unter sorgfältiger Prüfung die Eignung des Patienten eruiert warden ( $\bullet$ Abb. 1, 2).

\section{Vorbereitung präoperativ \\ $\nabla$}

Neben den allgemeinen chirurgischen präoperativen Vorbereitungen und einer exakten Fotodokumentation soll eine gründliche Inspektion und Untersuchung des zu behandelnden Areals im Hinblick auf Hernien, Einziehungen, Narben und Hautbeschaffenheit durchgeführt werden. Zudem soll das zur geplanten Rekonstruktion benötigte Volumen abgeschätzt und dafür infrage kommende Entnahmestellen bestimmt werden, wobei der Wunsch des Patienten und der Fettgewebsüberschuss wesentlich sein sollte. Bezüglich Zellqualität, Zusammensetzung und Überlebensrate ist kein Körperareal zu präferieren [1]. Ein Volumenschwund von 10 bis $50 \%$ soll bedacht werden, jedoch sollte aufgrund des Risikos von Fettgewebsnekrosen auf Überkorrekturen verzichtet werden. Für die Rekonstruktion größerer Volumenmengen sollte ein mehrzeitiges Vorgehen (Folgeeingriff frühestens nach 3 Monaten) gewählt werden.

\section{Durchführung der autologen Fetttransplantation $\nabla$}

Grundvoraussetzung ist die Durchführung durch einen Arzt, der die nötige Expertise bezüglich Lipoaspiration und Lipofilling aufweist, um die schonende Entnahme des Zelltransplantates zu gewährleisten. Entscheidend für ein optimales postoperatives Ergebnis ist die Entnahmetechnik. Gleichwertig bewertete Verfahren der Fettgewebsgewinnung sind:

- Technik nach Coleman [2-5]

- „Lipivage“ [6]

- Methode nach Shippert [7]

- Technik nach Khouri [8-10]

- Wasserstahl assistierte Verfahren [11]

Die wasserstrahlassistierte Liposuktion zeigt eine gute Qualität des Fetttransplantates mit sehr kleinen Partikeln, hoher Anzahl an Adipozyten und Stammzellen sowie minimalen Schäden an 


\section{Hauptindikationen}

Z.n. Brustkrebs und Brustrekonstruktion (Z.n. Mastektomie, Z.n. BET unter besonderer Beachtungen der Kontraindikationen)

- Brustassymmetrie nach Augmentation/Kapselkontraktur/Reduktion/Rekonstruktion [34]

- Komplettrekonstruktion mit Eigenfett

- Chronische Radio-/Strahlendermatitis (Stimulation der Neovaskularisation)

- Konturdefizite und Asymmetrien der Brust nach Radiatio [35, 36]

- Lappenplastiken zum Brustaufbau bei Z.n. Ablatio mammae in Verbindung mit Implantataugmentation [37]

Kongenitale und erworbene Brustfehlbildungen

- Mammahypoplasie, Mikromastie [35]

- Tuberöse/Tubuläre Brustdeformität [34,35]

- Polandsyndrom [34,35]

- Mamillenrekonstruktion [35,38]

- Pectus excavatum [39]

- Adhärente oder instabile Narben

- Deformität nach Mammareduktionsplastik oder Mastektomie [5, 35]

Ästhetisch-kosmetische Eingriffe und andere Erkrankungen:

- Volumenaufbau der Brust

- Z. n. Implantatentfernung zur Volumenaugmentation der Brust

- Deformität nach Mammaaugmentation

- Volumenaugmentation Gesicht-, Lippen-, Faltenkorrektur [41,42]

- Hemifacial Mikrosomie/Atrophie (Parry-Romberg-Syndrom) [43]

- Faciale Lipoatrophie durch Lupus erythematodes, HIV oder retrovirale Therapie [43,44]

- Hautrejuvenation

- Handverjüngung [35]

- Weichteilaugmentation an den Beinen [45]

- Gluteale Augmentation und Korrektur von Konturdeformitäten [45, 46]

- Genitalchirurgie/Transsexualität

- HNO (Stimmlippenaugmentation)

- Chronische Wunden [40]

- Zirkumskripte Sklerodermie/Coup-de-sabre-Läsionen [47]

- Weichteildefizite [45]

- Chronische Wunden [40]
Tab. 1 Indikationen der autologen Fetttransplantation.

\section{Kontraindikationen}

Absolute Kontraindikationen:

- Allgemein: Dysmorphophobie, unrealistische Erwartungshaltung, externe Motivation, u.a.

- Infrage stellen des Verfahrenserfolges durch lokale und systemische Erkrankungen

- Aktive maligne Grunderkrankung des Spender- oder Empfängersitus

- Schwangerschaft und Stillzeit

- Akute Infektionen im Spender- oder Empfängersitus

- Therapeutische Antikoagulation oder schwerwiegende Gerinnungsstörung

Besonders strenge Indikationsstellung:

- BRCA 1/2-Mutationen, positive Familienanamnese für Brustkrebserkrankungen [35]

- Nach BET eines DCIS oder MCA (Mucin-like Cancer associated Antigen) bei Rezidiv Freiheit frühestens 2 Jahre nach der lokalen Therapie unter kontrollierten Studienbedingungen

Strenge Indikationsstellung:

- Immunsupression

- Starker Nikotinabusus

Blut- und Lymphgefäßen sowie Nerven. Der Anteil intakter Fettzellen bei der wasserstrahlassistierten Methode kann bis zu $90 \%$ intakte Fettzellen enthalten. Andere Absaugmethoden weisen eine niedrigere Viabilitätsrate auf: unter Verwendung herkömmlicher Kanülen ohne ultraschall- oder wasserstrahlassistierter Liposuktion liegt die Zellviabilität bei $75-80 \%$, bei der Anwendung von laser-, ultraschall- oder radiofrequenz-assistierter Verfahren zeigt sich eine Viabilitätsrate von 30-50\% [12]. Um ein bestmöglichstes Ergebniss bezüglich Effizienz und Zellviabilität zu erhalten sollten bei der Lipoaspiration und Vorbereitung des Gewebes folgende Kriterien beachtet werden:

- Die Lipoaspiration soll unter Verwendung von Vakuumpumpen oder Spritzen erfolgen. Zu beachten ist ein kontinuierlicher Sog von $-0,5$ bis $-0,55$ bar, $[11,13]$. Sowohl abrupte Druckschwankungen z. B. durch ein Unterbrechen der Fettab- saugung als auch ein zu hoher Sog sollen vermieden werden, da sich dies negativ auf die Zellviabilität auswirkt (Shiffman and Mirrafati, 2001).

- Die Liposuktion soll in Tumeszenztechnik erfolgen. Die Tumeszenzlösung soll ein möglichst pH-neutrales, wenig zelltoxisches Lokalanästhetikum (CAVE Lidocain) und einen Vasokonstriktor (Adrenalin, 1:200000) enthalten [14-17]. Der Vasokonstriktor (z.B. Adrenalin) verringert die Blutbeimengungen im gewonnenen Gewebe.

- Spezielle, für die Lipoaspiration im Handel erhältliche Absaugkanülen sollen verwendet werden. Kanülen mit zu engen Lumina beeinflussen das Transplantatüberleben negativ. 


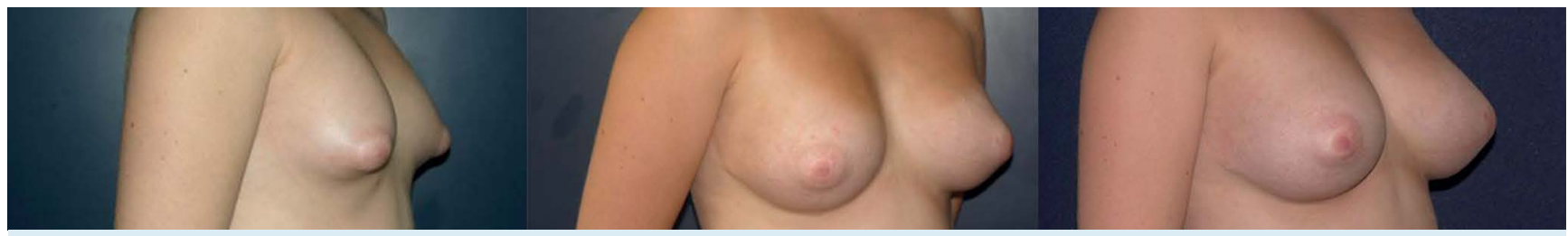

Abb. 1 19-jährige Patientin mit tubulärer Brustdeformität. Vor, 8 und 18 Monate nach Lipofilling mit 350 cc/Seite in zwei operativen Sitzungen.

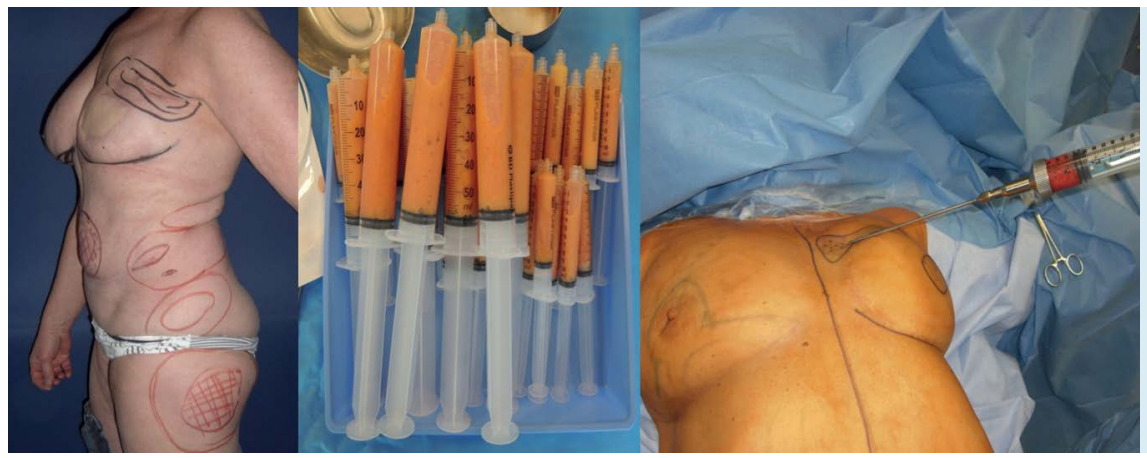

Abb. 2 Links: typische Entnahmestellen zur Fettgewinnung rot angezeichnet. Mitte: Menge gewonnenen Fettgewebes vor Transplantation. Rechts: Volumenaugmentation des oberen Brustquadranten zur Angleichung nach DIEP-Lappenplastik.

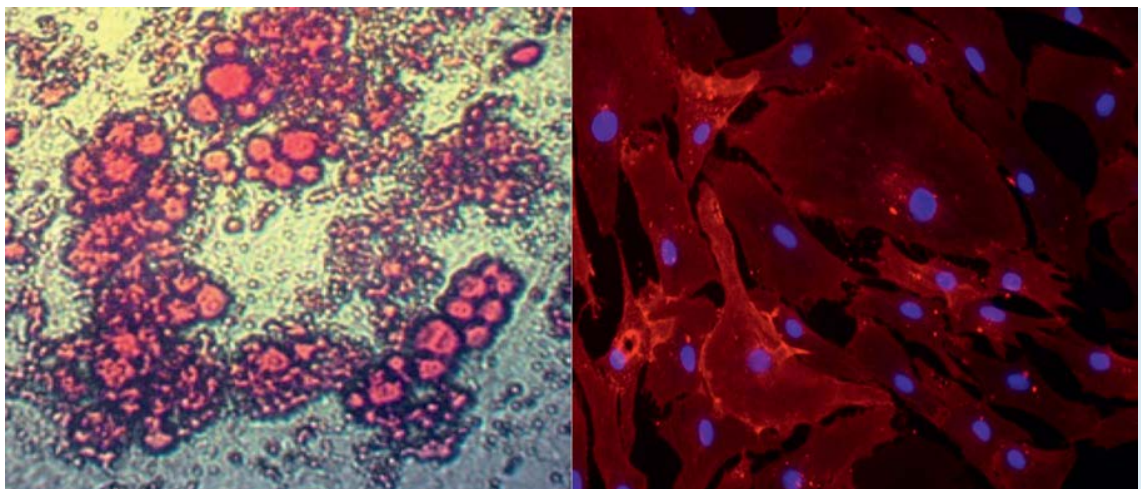

Abb. 3 Linkes Bild: Adipogene Differenzierung der ASCs in 200 x Vergrößerung, rechtes Bild: ASCs nach fluoreszierender (rot) Anfärbung u. DAPI Staining der Kerne, Vergrößerung $20 \mathrm{x}$.

- Geschlossene Systeme sind empfohlen, um die Exposition an der Luft und die bakterielle Kontamination möglichst gering zu halten [18]

- Die Liposuktion soll mit einer minimal-traumatischen Technik erfolgen. Ein zu oberflächliches Absaugen oder ein „Übersaugen" soll vermieden werden.

- Um das gewonnene Fettgewebe für die Transplantation vorzubereiten sollen die intakten Adipozyten und ASCs (adipose tissue-derived stem cells, mesenchymale Stammzellen des Fettgewebes) von Serum, Blut, Tumeszenzlösung, freiem Fett und avitalen Zellen getrennt werden. Dies kann durch Zentrifugation (CAVE Zeit, Zentrifugengröße und Umdrehungszahl), Filtration, Sedimentierung und Dekantierung erreicht werden.

\section{Lipoinjektion}

$\nabla$

Auch bei der Transplantation des gewonnenen und aufbereiteten Fettgewebes gibt es mehrere Faktoren zu beachten, damit gute Resultate erzielt werden.

- Stumpfe, gerade oder gebogene Luerlock-Kanülen mit einem Lumen von 1-3 mm und einer Öffnung sollten eingesetzt werden [19-22]. Spitze Kanülen bergen ein erhöhtes Komplikationsrisiko bezüglich intravasaler Injektionen, Blutungen und Verletzung von Nerven [23]. Jedoch können bei der Injek- tion von Eigenfett im Gesicht auch spitze Kanülen verwendet werden. Hier kommen in aller Regel Kanülen mit einem noch kleineren Lumen zum Einsatz.

- Über mehrere ca. $2 \mathrm{~mm}$ große Stichinzisionen mithilfe eines Stichskalpells oder einer dicken Hohlkanüle (z.B. 18Gauge) sollte das Fettgewebe unter Verwendung von Luerlock-Spritzen mit einem Füllvolumen von 1-20 ml injiziert werden. Das Spritzenvolumen richtet sich nach Empfängerareal, für das Gesicht sind 1-3 $\mathrm{ml}$ Spritzen und für die Gesäßregion bis zu $40 \mathrm{ml}$ Spritzen empfohlen. Das geringe Spritzenvolumen erlaubt einerseits die Kontrolle über Menge und Platzierung, andererseits werden weniger schädigende Drücke auf das Fettgewebe ausgeübt.

- Die Abgabe kleiner Portionen (0,50 ml, im Gesicht 0,02$0,1 \mathrm{ml}$ ) in verschiedene Schichten und fächerartig über mehrere Tunnel ist essentiell für die Einheilungsquote [24]. Beim Lipofilling der Brust ist die Injektion ins Drüsengewebe zu vermeiden.

- Nach aktuellem Wissenstand sollte das gewonnene Fettgewebe zeitnah in der gleichen Operationssitzung in das Zielgebiet injiziert werden. Aktuell liegen keine Standards bezüglich Aufbewahrung des Fettgewebes durch Einfrieren und späterer Verwendung nach Auftauen vor. 


\section{Postoperatives Management}

Abhängig vom behandelten Körperareal gibt es bestimmte Aspekte zu beachten und verschiedene Maßnahmen zu ergreifen. Im Gesicht kann ein leichtes Modellieren des Transplantates in Verbindung mit einer mäßigen Lymphdrainage unter Kühlung und adäquater Analgesie eingesetzt werden. Nach Fetttransplantation in die Brust gibt es Empfehlungen für einen modellierenden/elastischen Verband, jedoch sollte ein direkter Druck auf das transplantierte Gewebe z.B. durch das vorherige Anlegen von Watteverbänden vermieden werden. Im Bereich der unteren Extremität und des Gesäßes kann ein leichter Kompressionsverband und niedermolekulare Heparine zur Thromboseprophylaxe erwogen werden. Insgesamt ist bei Fetttransplantationen am Körperstamm und an der unteren Extremität eine körperliche Schonung je nach Ausmaß und Lokalisation der Absaugung zu befürworten, auf Massagen sollte verzichtet werden. Die Entnahmeareale sollten postoperativ mittels Kompressionsmieder versorgt werden.

Um Komplikationen (Hämatome, Infektionen, Nekrosen) frühestmöglich zu erkennen, soll regelmäßig und vor allem am ersten postoperativen Tag eine Kontrolle der Wundregionen stattfinden. Postoperative Verlaufskontrollen zur Erhebung von Quantität (Graftvolumen) und Qualität (Komplikationen) sollen durch den Vergleich der prä- und postoperativen Fotodokumentation und durch apparative Verfahren wie 3D-Oberflächenscan, MRT und Sonografie erfolgen.

\section{Gesetzliche Rahmenbedingungen \\ $\nabla$}

Die Herstellung und Anwendung zelltherapeutischer Präparate bzw. klinischer Prüfpräparate werden in Deutschland im Arzneimittelgesetzt (AMG) geregelt. Auf EU-Ebene bilden u.a. die Geweberichtlinie (RL/2004/23/EG) bzw. die direkt rechtlich verbindliche EU-Verordnung für neuartige Therapien (EG) Nr. 1394/2007 die Grundlage und fordern für den Einsatz zelltherapeutischer Präparate eine behördliche Genehmigung. In Deutschland sind im Gewebegesetz die EU-Regelungen ins AMG, Transplantations- gesetz (TPG) und Transfusionsgesetz (TFG) integriert worden. Ansprechpartner ist die für die Arzneimittelüberwachung zuständige Landesbehörde.

Autologes Fettgewebe wird aktuell von den Behörden nur dann als „klassische Gewebezubereitung“ eingestuft, wenn kein industrielles Verfahren zur Anwendung kommt, keine substanzielle Gewebeverarbeitung erfolgt ist und etablierte Herstellungsschritte eingehalten werden. Das prozessierte Fettgewebe kann als biotechnologisches Gewebeprodukt eingestuft werden (EG Nr. 1394/2007) und somit als Arzneimittel für neuartige Therapien (ATMP, advanced therapy medicinal products) deklariert werden, wenn diesem „Eigenschaften zur Regeneration, Wiederherstellung oder zum Ersatz menschlichen Gewebes“ zugeschrieben wird bzw. das Fettgewebe bei der therapeutischen Anwendung seine ursprüngliche Funktion nicht erfüllt.

Aufgrund der steigenden Komplexität und Anforderungen der gesetzlichen Regularien und Erlaubnisverfahren bietet das Paul-Ehrlich-Institut (PEI) Beratungsgespräche an, um für das jeweilige Produkt frühzeitig das Entwicklungsprogramm und den jeweils passenden regulatorischen Weg zu diskutieren.

\section{Patientensicherheit \\ $\nabla$}

Ein besonderes Augenmerk ist beim Fetttransfer den transplantierten ADSCs zu widmen $\bullet$ Abb. 3. Mesenchymale Fettstammzellen haben neben immunmodulatorischen Wirkungen ein regeneratives Potenzial bezüglich Proliferation, Zelldifferenzierung und Angiogenese. Essentiell für das Transplantatüberleben bei der autologen Fettransplantation ist die angiogene Wirkung der Zytokine, die von ASCs sezerniert werden [25-27]. Jedoch sollte dabei die in der Literatur beschriebene onkogene Wirkung von ASCs nicht vernachlässigt werden. In experimentellen in vivo und in vitro Arbeiten konnte das Risiko für lokales Tumorwachstum, insbesondere die onkogene Wirkung von Brustkrebszellen, gezeigt werden [28-32]. Das Auftreten eines lokalen oder systemischen Rezidives konnte in retrospektiven Studien bei Brustkrebspatientinnen mit oder ohne Lipofilling zur Brustrekonstruktion und bei Brustaugmentationen ohne Karzi-

Tab. 3 Zu beachtende Besonderheiten bei Brustrekonstruktion bei Z.n. MammaCa und Hochrisikopatienten.

Besonderheiten bei Mammakarzinompatientinnen und Hochrisikopatienten

- Leitliniengerechte Nachsorge

- Genaue Aufklärung über die potenzielle onkogene Wirkung der mittransplantierten ASCs besonders bei Hochrisikopatientinnen (positive Eigen- oder Familienanamnese, BRCA-1, BRCA-2, brusterhaltende Therapie)

- Bildgebende Verfahren (Sonografie, MRT, Mammografie) zur Erkennung von verdächtigen Läsionen und Mikrokalzifikationen

- Bei radiologisch ungeklärten Befunden Durchführen von Biopsien

- Ausgangsmammografie bzw. Sonografie zur Beurteilung des Verlaufs ab sechs Monate nach autologer Fetttransplantation

Tab. 4 Mögliche Komplikationen bei der autologen Fetttransplantation.

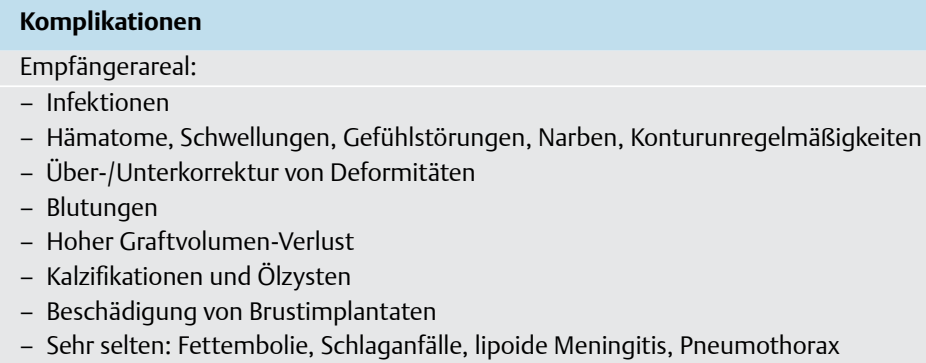

Spenderstelle:

- Ödeme, Hämatome, Serome

- Dysästhesien, Hypästhesien, Nervenläsionen

- Narbenbildung

- Konturunregelmäßigkeiten, Asymmetrien

- Hyper-/Hypopigmentation

- Unerwünschte Medikamentenreaktion, Juckreiz

- Infektbedingte Hautnekrosen

- Infektionen 
nomvorgeschichte im Vergleich zu Patientinnen mit anderen rekonstruktiven Eingriffen nach segmentaler oder totaler Mastektomie bisher nicht bestätigt werden. Wurde ein Lipofilling in einer gesunden Brust angewandt, zeigte sich nach 73 Monaten follow-up time kein Hinweis auf primären Brustkrebs. Erwähnenswert ist jedoch die in der Literatur beschriebene erhöhte Rezidivrate bei hormonrezeptor positiven Patientinnen mit Hormontherapie, die sich zur Rekonstruktion der Brust nach Brustkrebs einer autologen Fetttransplantation unterzogen haben [33] und ein erhöhtes Risiko für ein lokales Rezidiv bei vorangegangener intraepithelialer high grade Neoplasie [34]. Laut einer aktuellen retrospektiven Studie, in der Patientinnen zwischen 1981 und 2014 nach verschiedenen operativen Eingriffen bei malignen oder benignen Brusterkrankungen und anschließendem Lipofilling eingeschlossen wurden, wird die autologe Fetttransplantation zur Mammarekonstruktion jedoch als sicheres Verfahren eingestuft [33]. Dennoch ist bei rekonstruktiven Eingriffen nach Brustkrebs oder benignen Erkrankungen der Brust erhöhte Vorsicht und eine entsprechende Sorgfalt in der Nachsorge geboten. Stamm Zell angereicherte autologe Fetttransplantationen sollten zunächst nur im Rahmen klinisch, kontrollierter Studien durchgeführt werden. In $\odot$ Tab. 3 sind wichtige Aspekte bei Brustrekonstruktionen nach Brustkrebs und Hochrisikopatientinnen aufgeführt.

Wie bei jedem chirurgischen Eingriff bestehen auch bei der autologen Fetttransplantation Risiken und Komplikationen ( $\bullet$ Tab. 4). Deshalb ist eine Aufklärung des Patienten über mögliche Risiken und Komplikationen nach aktuellem Stand der Wissenschaft unverzichtbar.

Insgesamt ist die autologe Fetttransplantation für rekonstruktive und ästhetische Eingriffe nach aktuellem Stand der Wissenschaft und klinischen Erfahrungen aufgrund der niedrigen Komplikationsrate und des guten Outcomes bei korrekter Vorgehensweise und Durchführung der Operationstechnik unter penibler Sterilität als sicheres Therapieverfahren anzusehen. Jedoch sind weitere Studien und Untersuchungen nötig, um ungeklärte Aspekte näher zu erforschen und signifikante Aussagen treffen zu können.

\section{Interessenkonflikt: Nein.}

Institute

${ }^{1}$ Abteilung für Plastische, Hand- und Wiederherstellungschirurgie,

Universitätsklinikum Regensburg, Regensburg

${ }^{2}$ Klinik für Orthopädie, Unfall-, Hand- und Wiederherstellungschirurgie,

Plastische und Ästhetische Chirurgie sowie Verbrennungschirurgie,

Leverkusen

${ }^{3}$ Handchirurgie, Plastische Chirurgie und Ästhetische Chirurgie, Klinikum der Ludwig-Maximilians Universität (LMU) München, München

${ }^{4}$ Ente Ospedaliero Cantonale Cirurgia Plastica, ricostruttiva ed estetica Viganello, Lugano

5 Praxisklinik Kaiserplatz, Frankfurt am Main

${ }^{6}$ Klinik für Plastische und Ästhetische, Hand- und Wiederherstellungschirurgie, Caritas Krankenhaus St. Josef, Regensburg

Klinik für Plastische und Ästhetische Chirurgie im Sana Klinikum HamelnPyrmont, Oldenburg

${ }^{8}$ Klinik für Hand-, Plastische- und Rekonstruktive Chirurgie - Schwerbrandverletztenzentrum, BG-Unfallklinik Ludwigshafen, Ludwigshafen am Rhein ${ }^{9}$ Klinik für Plastische- und Handchirurgie, Universitätsklinikum Freiburg, Freiburg

${ }^{10}$ Klinik für Plastische Chirurgie und Handchirurgie, Klinikum rechts der Isar der Technischen Universität München, München

${ }^{11}$ Klinik für Plastische, Rekonstruktive und Ästhetische Chirurgie,

Handchirurgie, Klinikum Bremen Mitte, Bremen

${ }^{12}$ Haut- und Laserzentrum an der Oper, München

${ }^{13}$ Klinik für Plastische Chirurgie, Hand- und Verbrennungschirurgie, Uniklinik RWTH Aachen, Aachen
${ }^{14}$ Klinik für Plastische Chirurgie und Handchirurgie, Klinikum rechts der Isar der Technischen Universität München, München

${ }^{15}$ Klinik für Plastische und Ästhetische Chirurgie, St. Josefs-Krankenhaus Potsdam-Sanssouci, Potsdam

${ }^{16}$ Park-Klinik Birkenwerder, Birkenwerder

${ }^{17}$ Plastische Chirurgie, Charité - Universitätsmedizin Berlin, Berlin

${ }^{18}$ Marienhospital Düsseldorf, Düsseldorf

${ }^{19}$ DR-aesthetics, Köln

${ }^{20}$ Rosenparkklinik GmbH, Darmstadt

${ }^{21}$ Klinik für Plastische, Ästhetische, Hand- und Wiederherstellungschriurgie, Med. Hochschule Hannover, Hannover

${ }^{22}$ Plastische- und Handchirurgische Klinik, Universitätsklinikum Erlangen, Erlangen

\section{Literatur}

1 Rohrich RJ, Sorokin ES, Brown SA. In Search of improved fat transfer viability: a quantitative analysis of the role of centrifugation and harvest site. Plast Reconstr Surg 2004; 113: 391-395. doi:10.1097/01. PRS.0000097293.56504.00

2 Coleman SR. Structural fat grafts: the ideal filler? Clin Plast Surg 2001; 28: 111-119

3 Coleman SR. Hand rejuvenation with structural fat grafting. Plast Reconstr Surg 2002; 110: 1731-1744-1747. doi:10.1097/01.PRS.00 00033936.43357.08

4 Coleman SR. Structural fat grafting: more than a permanent filler. Plast Reconstr Surg 2006; 118: S108-S120. doi:10.1097/01. prs.0000234610.81672.e7

5 Coleman SR, Saboeiro AP. Fat grafting to the breast revisited: safety and efficacy. Plast Reconstr Surg 2007; 119: 775-785-787. doi:10.1097/01. prs.0000252001.59162.c9

6 Ferguson REH, Cui X, Fink BF et al. The viability of autologous fat grafts harvested with the LipiVage system: a comparative study. Ann Plast Surg 2008; 60: 594-597. doi:10.1097/SAP.0b013e31817433c5

7 Shippert RD. Autologous fat transfer: eliminating the centrifuge, decreasing lipocyte trauma and establishing standardization for scientific study. Ajcs-23-01-04 2127 - AFT-Stand 2006. http://192.185.101.132/ shippert/resources/wp-content/uploads/2014/09/AFT-standardizati on.pdf (accessed June 12, 2016)

8 Khouri RK, Khouri R-ER, Lujan-Hernandez JR et al. Diffusion and perfusion: the keys to fat grafting. Plast Reconstr Surg Glob Open 2014; 2: e220. doi:10.1097/GOX.0000000000000183

9 Khouri RK, Rigotti G, Cardoso E et al. Megavolume autologous fat transfer: part I. Theory and principles. Plast Reconstr Surg 2014; 133 : 550-557. doi:10.1097/01.prs.0000438044.06387.2a

10 Khouri RK, Rigotti G, Cardoso E et al. Megavolume autologous fat transfer: part II. Practice and techniques. Plast Reconstr Surg 2014; 133 : 1369-1377. doi:10.1097/PRS.0000000000000179

11 Ueberreiter K, von Finckenstein JG, Cromme F et al. BEAULITM - a new and easy method for large-volume fat grafts. Handchir Mikrochir Plast Chir Organ Deutschsprachigen Arbeitsgemeinschaft Für Handchir Organ Deutschsprachigen Arbeitsgemeinschaft Für Mikrochir Peripher Nerven Gefässe Organ Ver Dtsch Plast Chir 2010; 42: 379-385. doi:10.1055/s-0030-1267913

12 Sasaki GH. Body jet, reshapes future of grafting. aesthetic guide january February 2010 TAG Page 78 2010. http://digital.miinews.com/ publication/? $\mathrm{i}=32276 \& \mathrm{p}=78$ (accessed June 18,2016 )

13 Niechajev I, Sevćuk O. Long-term results of fat transplantation: clinical and histologic studies. Plast Reconstr Surg 1994; 94: 496-506

14 Keck $M$, Zeyda $M$, Gollinger $K$ et al. Local anesthetics have a major impact on viability of preadipocytes and their differentiation into adipocytes. Plast Reconstr Surg 2010; 126: 1500-1505. doi:10.1097/ PRS.0b013e3181 ef8beb

15 Keck M, Janke J, Ueberreiter K. Viability of preadipocytes in vitro: the influence of local anesthetics and pH. Dermatol Surg Off Publ Am Soc Dermatol Surg Al 2009; 35: 1251-1257 doi:10.1111/j.15244725.2009.01220.x

16 Sasaki GH. Water-assisted liposuction for body contouring and lipoharvesting: safety and efficacy in 41 consecutive patients. Aesthetic Surg J Am Soc Aesthetic Plast Surg 2011; 31: 76-88. doi:10.1177/1090820 × 10391465

17 Sommer B, Sattler G. Current concepts of fat graft survival: histology of aspirated adipose tissue and review of the literature. Dermatol Surg Off Publ Am Soc Dermatol Surg Al 2000; 26: 1159-1166

18 Largo RD et al. Autologe Fetttransplantation in die weibliche Brust 2011-28-150.pdf 2011. http://www.medicalforum.ch/docs/smf/ archiv/de/2011/2011-28/2011-28-150.pdf (accessed June 11, 2016)

19 Donofrio LM. Techniques in facial fat grafting. Aesthetic Surg J Am Soc Aesthetic Plast Surg 2008; 28: 681-687. doi:10.1016/j.asj.2008.09.003 
20 Guijarro-Martínez R, Alba LM, Mateo MM et al. Autologous fat transfer to the cranio-maxillofacial region: Updates and controversies. J Craniomaxillofacial Surg 2011; 39: 359-363. doi:10.1016/j. jcms.2010.07.004

21 Rosing JH, Wong G, Wong MS et al. Autologous fat grafting for primary breast augmentation: a systematic review. Aesthetic Plast Surg 2011; 35: 882-890. doi:10.1007/s00266-011-9691-9692

22 Sarfati I, Ihrai T, Kaufman $G$ et al. Adipose-tissue grafting to the post-mastectomy irradiated chest wall: preparing the ground for implant reconstruction. J Plast Reconstr Aesthetic Surg JPRAS 2011; 64: 1161-1166. doi:10.1016/j.bjps.2011.03.031

23 Obagi S. Autologous fat augmentation for addressing facial volume loss. Oral Maxillofac Surg Clin N Am 2005; 17: 99-109. vii. doi:10.1016/j. coms.2004.11.001

24 Bucky LP, Percec I. The science of autologous fat grafting: views on current and future approaches to neoadipogenesis. Aesthetic Surg J Am Soc Aesthetic Plast Surg 2008; 28: 313-321-324 doi:10.1016/j. asj.2008.02.004

25 Condé-Green A, Baptista LS, de Amorin NFG et al. Effects of centrifugation on cell composition and viability of aspirated adipose tissue processed for transplantation. Aesthet Surg J 2010; 30: 249-255. doi: $10.1177 / 1090820 \times 10369512$

26 Gehmert S, Gehmert S, Hidayat M et al. Angiogenesis: the role of PDGFBB on adipose-tissue derived stem cells (ASCs). Clin Hemorheol Microcirc 2011; 48: 5-13. doi:10.3233/CH-2011-1397

27 Prantl L, Heine N. Options for regenerative therapy in the field of breast surgery. Handchir Mikrochir Plast Chir Organ Deutschsprachigen Arbeitsgemeinschaft Für Handchir Organ Deutschsprachigen Arbeitsgemeinschaft Für Mikrochir Peripher Nerven Gefässe Organ Ver Dtsch Plast Chir 2012; 44: 103-111. doi:10.1055/s-0031-1297966

28 Massa M, Gasparini S, Baldelli I et al. Interaction between breast cancer cells and adipose tissue cells derived from fat grafting. Aesthetic Surg J Am Soc Aesthetic Plast Surg 2016; 36: 358-363. doi:10.1093/ asj/sjv194

29 Kamat $P$, Schweizer $R$, Kaenel $P$ et al. Human adipose-derived mesenchymal stromal cells may promote breast cancer progression and metastatic spread. Plast Reconstr Surg 2015; 136: 76-84. doi:10.1097| PRS.0000000000001321

30 Pallua N, Paul NE, Burghardt B et al. Adipose tissue increases the proliferation of melanoma cell lines in vitro. J Craniofac Surg 2015; 26 : 1403-1407. doi:10.1097/SCS.0000000000001593

31 Yu JM, Jun ES, Bae YC et al. Mesenchymal stem cells derived from human adipose tissues favor tumor cell growth in vivo. Stem Cells Dev 2008; 17: 463-474. doi:10.1089/scd.2007.0181

32 Weigand A, Boos AM, Tasbihi $K$ et al. Selective isolation and characterization of primary cells from normal breast and tumors reveal plasticity of adipose derived stem cells. Breast Cancer Res BCR 2016; 18. doi:10.1186/s13058-016-0688-2

33 Kronowitz SJ, Mandujano CC, Liu J et al. Lipofilling of the breast does not increase the risk of recurrence of breast cancer: A Matched controlled study. Plast Reconstr Surg 2016; 137: 385-393 doi:10.1097/01. prs.0000475741.32563.50

34 Del Vecchio DA, Bucky LP. Breast augmentation using preexpansion and autologous fat transplantation: a clinical radiographic study. Plast Reconstr Surg 2011; 127: 2441-2450. doi:10.1097| PRS.0b013e3182050a64
35 Gutowski KA, ASPS Fat Graft Task Force. Current applications and safety of autologous fat grafts: a report of the ASPS fat graft task force. Plast Reconstr Surg 2009; 124: 272-280. doi:10.1097/PRS. 0b013e3181a09506

36 Rennekampff $\mathrm{H}-\mathrm{O}$, Reimers $\mathrm{K}$, Gabka CJ et al. Current perspective and limitations of autologous fat transplantation - "consensus meet ing" of the German Society of Plastic, Reconstructive and Aesthetic Surgeons at Hannover; September 2009. Handchir Mikrochir Plast Chir Organ Deutschsprachigen Arbeitsgemeinschaft Für Handchir Organ Deutschsprachigen Arbeitsgemeinschaft Für Mikrochir Peripher Nerven Gefässe Organ Ver Dtsch Plast Chir 2010; 42: 137-142. doi:10.1055/s-0030-1249672

37 Missana MC, Laurent I, Barreau L et al. Autologous fat transfer in reconstructive breast surgery: indications, technique and results. Eur J Surg Oncol J Eur Soc Surg Oncol Br Assoc Surg Oncol 2007; 33: 685-690. doi:10.1016/j.ejso.2006.12.002

38 Delaporte T, Delay E, Toussoun $G$ et al. Breast volume reconstruction by lipomodeling technique: about 15 consecutive cases. Ann Chir Plast Esthét 2009; 54: 303-316 doi:10.1016/j.anplas.2008.11.007

39 Tiryaki T, Findikli N, Tiryaki D. Staged stem cell-enriched tissue (SET) injections for soft tissue augmentation in hostile recipient areas: a preliminary report. Aesthetic Plast Surg 2011; 35: 965-971. doi:10.1007/ s00266-011-9716-x

40 Rigotti G, Marchi A, Galiè M et al. Clinical treatment of radiotherapy tissue damage by lipoaspirate transplant: a healing process mediated by adipose-derived adult stem cells. Plast Reconstr Surg 2007; 119: 1409-1422-1424. doi:10.1097/01.prs.0000256047.47909.71

41 Guyuron B, Majzoub RK. Facial augmentation with core fat graft: a preliminary report. Plast Reconstr Surg 2007; 120: 295-302. doi:10.1097/01.prs.0000264399.40701.71

42 Moseley TA, Zhu M, Hedrick MH. Adipose-derived stem and progenitor cells as fillers in plastic and reconstructive surgery. Plast Reconstr Surg 2006; 118: S121-S128. doi:10.1097/01.prs.0000234609.74811.2e

43 Yoshimura $K$, Sato $K$, Aoi $N$ et al. Cell-assisted lipotransfer for facial lipoatrophy: efficacy of clinical use of adipose-derived stem cells. Dermatol Surg Off Publ Am Soc Dermatol Surg Al 2008; 34: 1178-1185. doi:10.1111/j.1524-4725.2008.34256.x

44 Burnouf $M$, Buffet $M$, Schwarzinger $M$ et al. Evaluation of Coleman lipostructure for treatment of facial lipoatrophy in patients with human immunodeficiency virus and parameters associated with the efficiency of this technique. Arch Dermatol 2005; 141: 1220-1224. doi:10.1001/archderm.141.10.1220

45 de Pedroza $L V$. Fat transplantation to the buttocks and legs for aesthetic enhancement or correction of deformities: long-term results of large volumes of fat transplant. Dermatol Surg Off Publ Am Soc Dermatol Surg Al 2000; 26: 1145-1149

46 Pereira LH, Sterodimas A. Macroscopic and microscopic proof of longterm survival of gluteal fat transplantation. Plast Reconstr Surg 2009; 123: e162-e163. doi:10.1097/PRS.0b013e31819e5d59

47 Consorti G, Tieghi R, Clauser LC. Frontal linear scleroderma: long-term result in volumetric restoration of the fronto-orbital area by structural fat grafting. J Craniofac Surg 2012; 23: e263-e265 doi:10.1097/ SCS.0b013e31824ef7e8 